\title{
Substantiation Stages of Rehabilitation at the Patients with Metaepiphyseal Osteomyelitis
}

\author{
Lukianenko Dmytro Mykolaiovych \\ Dnipropetrovsk Medical Academy Ministry of Health of Ukraine, Dnipro, Ukraine
}

\section{Email address:}

childsurgery@ukr.net

\section{To cite this article:}

Lukianenko Dmytro Mykolaiovych. Substantiation Stages of Rehabilitation at the Patients with Metaepiphyseal Osteomyelitis. American Journal of Pediatrics. Vol. 3, No. 5, 2017, pp. 46-50. doi: 10.11648/j.ajp.20170305.14

Received: May 3, 2017; Accepted: May 22, 2017; Published: October 18, 2017

\begin{abstract}
Surgeons and orthopedists, which carried out medical and rehabilitative measures at the patients with metaepiphyseal osteomyelitis did not pay attention to the socio-psychological rehabilitation. Purpose of research is determination volume of methods for rehabilitation this group of patients. In our study we carried out sociological survey, using own questionnaire. On the basic question of the questionnaire - does the disease influence to the realization of vital important plans in a patient's life, $8(15.1 \%)$ respondents had been answered, that osteomyelitis destroyed all plans in their life. The given conclusion was shown primary among male - patients $-18.2 \%$, than among female - only $10 \%(\mathrm{p}>0.05)$. Volume of rehabilitation we combined with issues of abilitation - the system of therapeutic measures, which should prevent and eliminate pathological conditions at the children with metaepiphyseal osteomyelitis.
\end{abstract}

Keywords: Children, Metaepiphyseal Osteomyelitis, Medical Rehabilitation, Abilitation

\section{Introduction}

Review of the numerous studies [1-8] demonstrated that acute clavicle osteomyelitis in children is representing in less $<3 \%$ of osteomyelitis cases. Acute clavicle osteomyelitis mainly affects older children and has generally good prognosis. Staphylococcus aureus is most commonly implicated and surgery may be needed $[9,10,11]$.

Osteomyelitis is inflammation of bones located in the metaphysis and is more frequent in the lower limbs [12]. The diagnosis of osteomyelitis in childhood is usually straightforward and timely use of appropriate antimicrobial therapy has virtually eliminated mortality [13]. In $65-75 \%$ of cases the femur, tibia, or humorous is involved. Involvement of other long bones is less common and of bones such as clavicles, ribs, spine, and bones of the hands and feet is unusual; thus, at these sites, diagnostic problems may present [14]. Clavicle is involved in 1-3\% [15].

A total of 89 articles were retrieved from literature search, which $[16,17,18,19]$ reported 16 cases of acute clavicle osteomyelitis in children and adolescents (ages ranging between 0 and 16 years) that were included in the analysis.

Infection after surgical treatment of fractures is a complication with significant morbidity and in rare cases even mortality [20]. Consequences of infections include delayed or non-union of the fracture [21].

Most research in this field focuses on peri-prosthetic infection, despite of the different treatment challenges in prosthetic surgery and osteosynthesis. Treatment algorithms have been developed, which dictate aggressive debridement, antibiotic treatment, and if necessary staged replacement of the prosthetic material [22].

Majority of articles is devoted to the bone and joint infections in children and acute hematogenous osteomyelitis [23]. Acute hematogenous osteomyelitis (AHO) is one of the commonest bone infections in childhood. The main clinical symptom and sign in AHO is pain and tenderness over the affected bone especially in the metaphyseal region [24].

The study [25] shows that acute osteomyelitis of the clavicle tends to affect older children, as in our case, in which the patient was a 12-year-old boy. Recurrence happened in 1/16 cases and persistence of symptoms happened in 2/16 cases.

Wang X. et al. [26] considered, that chronic hematogenous osteomyelitis often results from the improper treatment of acute hematogenous osteomyelitis. As a rule, the complications of chronic hematogenous tibia osteomyelitis treated with the induced membrane technique.

A collective of authors [27] proved that acute clavicle 
osteomyelitis in children is rare representing $<3 \%$ of osteomyelitis cases. Osteomyelitis was hematogenous in most cases, with $S$. aureus being the most frequent cause, isolated from either blood or tissue. Acute clavicle osteomyelitis mainly affects older children and has generally good prognosis.

In the research of Morita M. et al. [28] had been revealed various conditions, including bacterial infection, which can promote osteonecrosis: following invasive dental therapy with anti-bone resorptive agents, some patients develop osteonecrosis in the jaw; however, pathological mechanisms underlying these outcomes remain unknown.

Wagner JM. et al. [29] study osteomyelitis as a frequent consequence of open fractures thus representing a common bone infection with subsequent alteration of bone regeneration. Impaired bone homeostasis provokes serious variations in the bone remodeling process, thereby involving multiple inflammatory cytokines to activate bone healing.

Tuck M. et al [30] described a patient with ALK-negative ALCL presenting with clinical and radiographic findings suggesting osteomyelitis 6 months after left rotator cuff repair surgery. ALCL should be considered in patients not responding to therapies for osteomyelitis.

In the case study [31], 66-year-old Caucasian female presented with insidious sciatic pain leading to an uncommon diagnosis of tuberculous (TB) osteomyelitis with unknown portal entry. Considering TB in the differential diagnosis of a 'bone abscess', it is of paramount importance to come to a correct diagnosis.

At work [32] was study acute hematogenous osteomyelitis (AHO) in children as an ideal condition due to its representation of a wide spectrum of disorders that comprise pediatric musculoskeletal infection. Proper care for children with AHO is multidisciplinary and collaborative.

In the research [33] was demonstrated a case of a man aged 68 years presenting to the emergency department with a 3-day history of fever, low back, right hip and leg pain. It was diagnosed Staphylococcus aureus vertebral osteomyelitis complicated by recurrent epidural abscess and severe sepsis.

Hellebrekers P. et al. [34] considered, that infection after osteosynthesis is an important complication with significant morbidity and even mortality. The authors analyzed the effect of such an aggressive standardized treatment regime with implant retention for acute, existing $<3$ weeks, infection after osteosynthesis.

Hudson JW. et al. [35] research the response of mandibular osteomyelitis treated by surgical decortication with disruption of the affected adjacent periosteum in concert with long-term targeted antibiotic therapy. The hypothesis is that, by removing the buccal cortical plate and disrupting the hypertrophically inflamed adjacent periosteum, the medullary bone will be brought in contact with bleeding tissue and circulating immunologic factors and antibiotics, which will promote definitive resolution.

In Ukraine surgeons and orthopedists carried out medical and rehabilitative measures at the patients with metaepiphyseal osteomyelitis (MEO) with the purpose of physical recovery. At the same time, the given patients need firstly the social and psychological rehabilitation. Solution of these problems has a great medico-social significance.

Purpose of the study is to determine volume of methods for medical rehabilitation of the patients with MEO.

\section{Materials and Methods}

Research was conducted with using sociological survey by a specially designed questionnaire. Questionnaire included questions, which were focused on the socio-psychological and physical condition of the patients with MEO. We investigated contingent of persons (53 respondents), who were treated in the clinic 20 years ago with severe complications and consequences of MEO.

\section{Results and Discussion}

The conducted rehabilitation in childhood age and in the long terms after diseases, respondents estimated as not in the full volume (table 1). Among these respondents, 28 (52.8\%) patients were not received any treatment after discharge from the hospital and in the polyclinic. Treatment in the hospital received only $11(20.8 \%)$ respondents, sanatorium and resort treatment received $13(24.5 \%)$ of patients with MEO.

Table 1. Rehabilitation of a primary disease among the respondents.

\begin{tabular}{|c|c|c|c|c|c|c|}
\hline \multirow{2}{*}{ Frequency of supervision and treatment } & \multicolumn{2}{|c|}{ In the polyclinic } & \multicolumn{2}{|c|}{ In the hospital } & \multicolumn{2}{|c|}{ Sanatorium-and -resort treatment } \\
\hline & absolute & $\%$ & absolute & $\%$ & absolute & $\%$ \\
\hline 1-2 times a year & 9 & 17.0 & 2 & 3.8 & 4 & 7.6 \\
\hline 2-3 times a year & 5 & 9.4 & 1 & 1.9 & - & - \\
\hline 3-4 times a year & 2 & 3.8 & - & - & - & - \\
\hline Once in a few years & 3 & 5.7 & - & - & 4 & 7.6 \\
\hline Unable to specify a term & 6 & 11.3 & 8 & 15.1 & 5 & 9.4 \\
\hline Not treated & 28 & 52.8 & 42 & 79.2 & 40 & 75.5 \\
\hline
\end{tabular}

To the main question of the questionnaire - does the disease influence on the implementation of life plans 8 (15.1\%) of respondents reported, that osteomyelitis has crossed out all their plans for life (table 2). This conclusion often had done the male patients $18.2 \%$ against $10 \%$ of female $(p>0.05)$. The given results were presented in
(Figure 1).

The basic conclusion of the given research is inadequate and unsystematic medical rehabilitation, which was determined during investigation of the patients' outpatient records. Anybody from the respondents did not ask for the help of psychologists. 


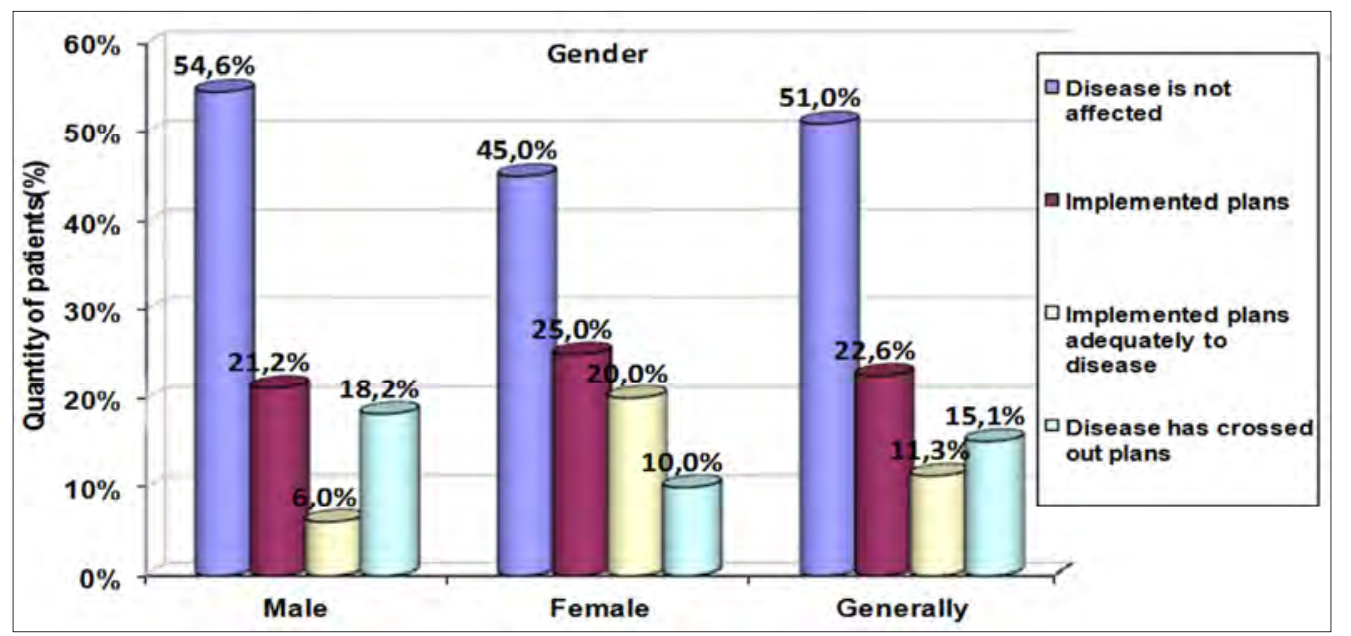

Figure 1. Graphical presentation of realization plans for life for patients with MEO.

Table 2. Distribution of the respondents' answers, depending on the gender and realization of life plans.

\begin{tabular}{|c|c|c|c|c|c|c|}
\hline \multirow{3}{*}{ Implementation of life plans } & \multicolumn{4}{|l|}{ Gender } & \multirow{2}{*}{\multicolumn{2}{|c|}{ Generally }} \\
\hline & \multicolumn{2}{|l|}{ male } & \multicolumn{2}{|l|}{ female } & & \\
\hline & absolute & $\%$ & absolute & $\%$ & absolute & $\%$ \\
\hline Disease is not affected & 18 & 54.6 & 9 & 45.0 & 27 & 51.0 \\
\hline Implemented plans & 7 & 21.2 & 5 & 25.0 & 12 & 22.6 \\
\hline Implemented plans adequately to the disease & 2 & 6.0 & 4 & 20.0 & 6 & 11.3 \\
\hline Disease has crossed out plans & 6 & 18.2 & 2 & 10.0 & 8 & 15.1 \\
\hline Generally & 33 & 100 & 20 & 100 & 53 & 100 \\
\hline
\end{tabular}

Note. Difference between the distribution of respondents' answers by gender was not statistically significance $\left(p=0.393, \chi^{2}=2.99\right)$.

Taking into account results of our research, rehabilitation of patients should begin in the acute period, during treatment of the complications, correction of the consequences. Psychological adaptation is necessary at the presence of a long-term corrected state of discomfort.

Results of research have been shown that all children, coming to the clinic with the diagnosis of metaepiphyseal osteomyelitis, should carry out a complex treatment, which allowed $93 \%$ of patients to decrease an inflammatory process. The patient undergoes an inpatient treatment for 1014 days, after that in the polyclinic conditions the restorative therapy and dynamic supervision till 2 months should be performing. Hereafter, a pathological process in the bone was estimated in the clinic, correction of the immobilization is carried out, if necessary - treatment of the disease recurrence. After 4-6 months of the acute process relief or later, almost all children, depending on the age (after 3 years), were recommended treatment of bone in the sanatorium, which have to specialized on the bone and joint system. The basic indications for sanatorium-resort treatment, should be violations of the bone structure, which leads to the orthopedic complications and consequences, i.e. disability. During 2 years the child should be under the supervision of the pediatric surgeon and orthopedist in the polyclinic. Recovery of the patient is evaluated not only on the form and function of the extremities restoration, but primary - on the restoration of the bone structure. Observation of the patient, treatment of probable consequences of the disease should be carried out by orthopedists - traumatologist.

Since 2005 all children, suffering from MEO, coming to the polyclinic of the Dnipropetrovsk Regional Children Clinical Hospital in Dnipro city should be covered with the consultation of psychologist. The psychologist's work is carried out with children, who have orthopedic consequences of MEO, which allows to prepare children for the life and work, taking accounts these consequences.

Scientifically proved a scheme of rehabilitation patients with MEO, because MEO - is acute disease, which requires the immediate medical and surgical intervention as well as in a case of acute appendicitis. Firstly, all this facts determines that the doctor's tactics should be focused on a source of infection, elimination causative agent of inflammation, prevention of the bones' structures destruction and damage of the cartilage surfaces of the joints. Therefore, volume of rehabilitation we combine with modern issues of abilitation, which means system of medical measures, which should prevent and eliminate pathological state at the children of early age, patients with MEO.

Scheme of the proposed methods of medical rehabilitation is presented in the graphical structure (figure 2).

\section{Conclusion}

In the article was scientifically proved the following stages of rehabilitation at the children with metaepiphyseal osteomyelitis: treatment of metaepiphyseal osteomyelitis in the acute period and clinical supervision by the surgeon and 
orthopedist up to 2 years (stage of rehabilitation); recovery of the structure and function of affected segment of a limb during growth of the patient (stage of clinical observation and correction by orthopedist); last stage - correction consequences of the metaepiphyseal osteomyelitis. Primary, metaepiphyseal osteomyelitis is developed at the result of severe defects, which closely connected with correction of the psychological state of children, which allowed patients to create adaptation to the society (during whole period of life in the orthopedist and psychologist).

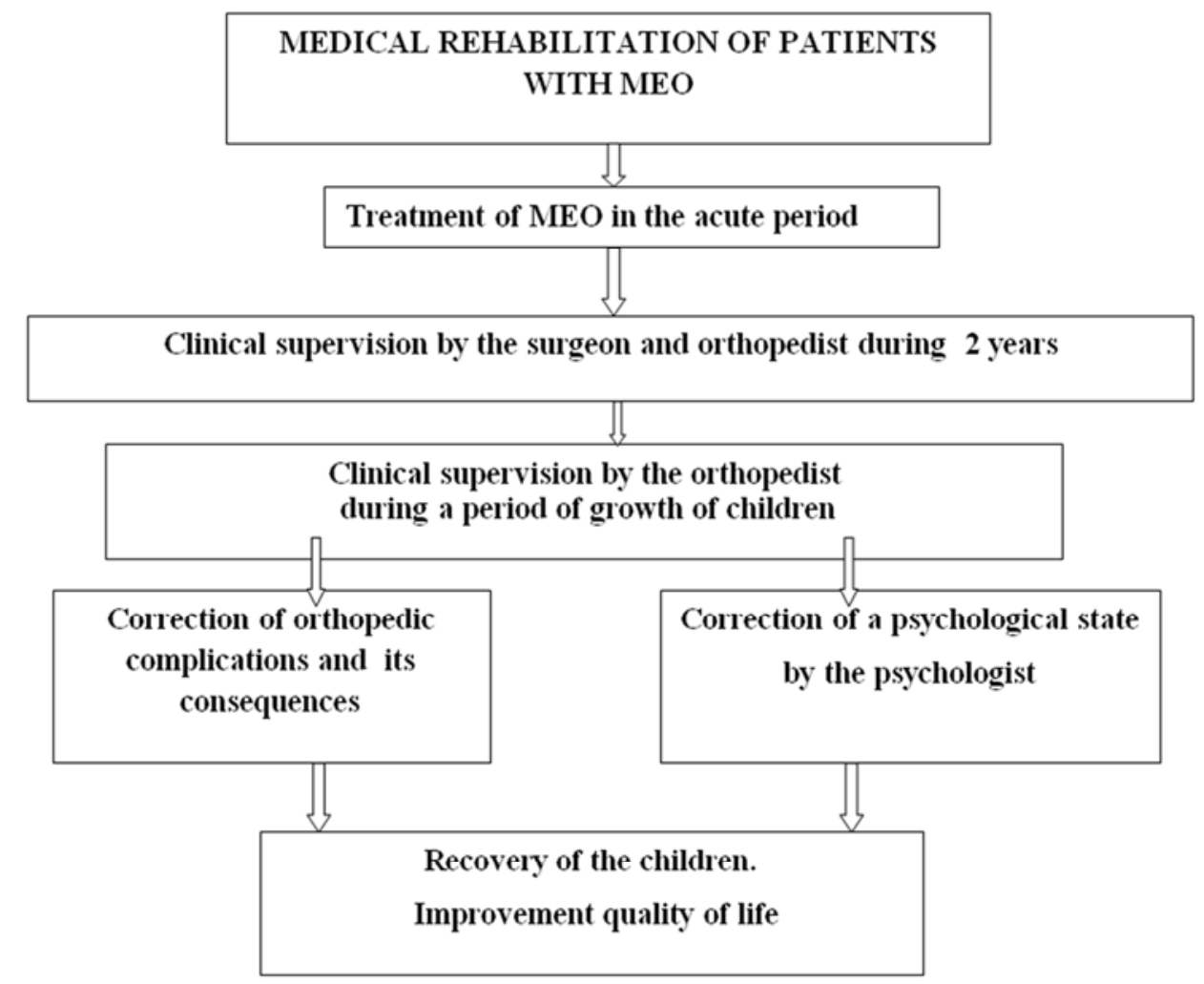

Figure 2. Scheme of medical rehabilitation of patients with metaepiphyseal osteomyelitis.

\section{References}

[1] Lowden C. M., Walsh S. J. Acute staphylococcal osteomyelitis of the clavicle. Journal of Pediatric Orthopaedics. 1997; 17 (4): 467-469.

[2] McGraw M. A., Mehlman C. T., Lindsell C. J., Kirby C. L. Postnatal growth of the clavicle: birth to 18 years of age. Journal of Pediatric Orthopaedics. 2009; 29 (8): 937-943.

[3] Moseley H. F. The clavicle: its anatomy and function. Clinical Orthopaedics and Related Research. 1968; 58: 17-27.

[4] Ogden J. A., Conlogue G. J., Bronson M. L. Radiology of postnatal skeletal development. III. The clavicle.Skeletal Radiology. 1979; 4 (4): 196-203.

[5] Gerscovich E. O., Greenspan A. Osteomyelitis of the clavicle: clinical, radiologic, and bacteriologic findings in ten patients. Skeletal Radiology. 1994; 23 (3): 205-210.

[6] Franklin J. L., Parker J. C., King H. A. Nontraumatic clavicle lesions in children. Journal of Pediatric Orthopaedics. 1987; 7 (5): 575-578.

[7] Morrey B. F., Bianco A. J., Jr. Hematogenous osteomyelitis of the clavicle in children. Clinical Orthopaedics and Related Research. 1977; (125): 24-28.

[8] Srivastava K. K., Garg L. D., Kochhar V. L. Osteomyelitis of the clavicle. Acta Orthopaedica Scandinavica. 1974; 45 (5): 662-667.

[9] Kocher M. S., Lee B., Dolan M., Weinberg J., Shulman S. T. Pediatric orthopedic infections: early detection and treatment. Pediatric Annals. 2006; 35 (2): 112-122.

[10] Pineda C., Vargas A., Rodríguez A. V. Imaging of osteomyelitis: current concepts. Infectious Disease Clinics of North America. 2006; 20(4): 789-825.

[11] Schuppen J., van Doorn M. M. A. C., van Rijn R. R. Childhood osteomyelitis: imaging characteristics.Insights into Imaging. 2012; 3 (5): 519-533.

[12] Robben S. G. Ultrasonography of musculoskeletal infections in children. European Radiology, Supplement. 2004; 14 (4): 65-77.

[13] Lew P. D. P., Waldvogel P. F. A. Osteomyelitis. Lancet. 2004; 364 (9431): 369-379.

[14] Peltola H., Pääkkönen M. Acute osteomyelitis in children. New England Journal of Medicine.2014; 370 (4): 352-360.

[15] Donovan R. M., Shah K. J. Unusual sites of acute osteomyelitis in childhood. Clinical Radiology.1982; 33 (2): 222-230.

[16] Darouiche RO. Treatment of infections associated with surgical implants. N Eng J Med. 2004; 350: 1422-9. 
[17] Trampuz A, Widmer AF. Infections associated with orthopedic implants. Curr Opin Infect Dis. 2006; 19: 349-56.

[18] Parvizi J, Adeli B, Zimistowski B, Restepo C, Greenwald AS. Management of periprosthetic joint infection: current knowledge: AAOS exhibit selection. J Bone Joint Surg. 2012; 94:104.

[19] Zimmerli W, Trampuz A, Oschner PE. Prosthetic-joint infections. N Engl J Med. 2004; 351: 1645-54.

[20] Worlock P, Slack R, Harvey L, Mawhinney R. The prevention of infection in open fractures: an experimental study of the effect of fracture stability. Injury. 1994; 25 (1): 31-38.

[21] Perren SM. Physical and biological aspects of fracture healing with special reference to internal fixation. Clin Orthop Relat Res. 1979; 138: 175-96.

[22] Wehrli W. Rifampin: mechanisms of action and resistance. Rev Infect Dis. 1983; 5(Suppl 3): S407-11.

[23] Stojicic S, Shen Y, Haapasalo M. Effect of the source of biofilm bacteria, level of biofilm, maturation, and type of disinfecting agent on the susceptibility of biofilm bacteria to antibacterial agents. J Endo. 2013; 39 (4): 473-7.

[24] Osmon DR, Berbari EF, Berendt AR, Lew D, Zimmerli W, Steckelberg JM, et al. Diagnosis and management of prosthetic joint infection: clinical practice guidelines by the infectious diseases society of America. Clin Infect Dis. 2013; 56(1): e1-25.

[25] Kiedrowski MR, Horswill AR. New approaches for treating staphylococcal biofilm infections. Ann N Y Acad Sci. 2011; 1241: 104-21.

[26] Wang X, Wang Z, Fu J. Induced membrane technique for the treatment of chronic hematogenous tibia osteomyelitis. BMC Musculoskelet Disord. 2017 Jan 23; 18(1): 33.

[27] Chrysochoou EA, Antachopoulos C, Badekas K, Roilides E. A Rare Case of Clavicle Osteomyelitis in a Child and Literature Review. Case Rep Pediatr. 2016; 2016: 82(52): 38.
[28] Morita M, Iwasaki R, Sato Y. et al. Elevation of proinflammatory cytokine levels following anti-resorptive drug treatment is required for osteonecrosis development in infectious osteomyelitis. Sci Rep. 2017 Apr 7; 7: 46322.

[29] Wagner JM, Jaurich H, Wallner C, et al. Diminished bone regeneration after debridement of posttraumatic osteomyelitis is accompanied by altered cytokine levels, elevated B cell activity, and increased osteoclast activity. J Orthop Res. 2017 Mar 6. doi: 10.1002/jor.23555.

[30] Tuck M, Lim J. et al. Anaplastic large cell lymphoma masquerading as osteomyelitis of the shoulder: an uncommon presentation. BMJ Case Rep. 2016 Dec 21; 2016. doi: 10.1136/bcr-2016-217317.

[31] De Mulder P, Harth C, Ide L, Vallaeys J, Baelde N, De Bo T. An uncommon cause of sciatic pain: tuberculous osteomyelitis of the ischial tuberosity. Acta Clin Belg. 2017 Jan 11:1-4. doi: 10.1080/17843286.2016.1271499.

[32] Funk SS, Copley LA. Acute Hematogenous Osteomyelitis in Children: Pathogenesis, Diagnosis, and Treatment. Orthop Clin North Am. 2017 Apr;48(2):199-208. doi: 10.1016/j.ocl.2016.12.007.

[33] Dunphy L, Iyer S, Brown C. Rare cause of back pain: Staphylococcus aureus vertebral osteomyelitis complicated by recurrent epidural abscess and severe sepsis. BMJ Case Rep. 2016 Dec 13;2016. pii: bcr2016217111. doi: 10.1136/bcr2016-217111.

[34] Hellebrekers P, Leenen LP, Hoekstra M, Hietbrink F. Effect of a standardized treatment regime for infection after osteosynthesis. J Orthop Surg Res. 2017 Mar 9;12(1):41. doi: 10.1186/s13018-017-0535-x.

[35] Hudson JW, Daly AP, Foster M. Treatment of Osteomyelitis: A Case for Disruption of the Affected Adjacent Periosteum. $J$ Oral Maxillofac Surg. 2017 Mar 18. doi: 10.1016/j.joms.2017.03.008. 